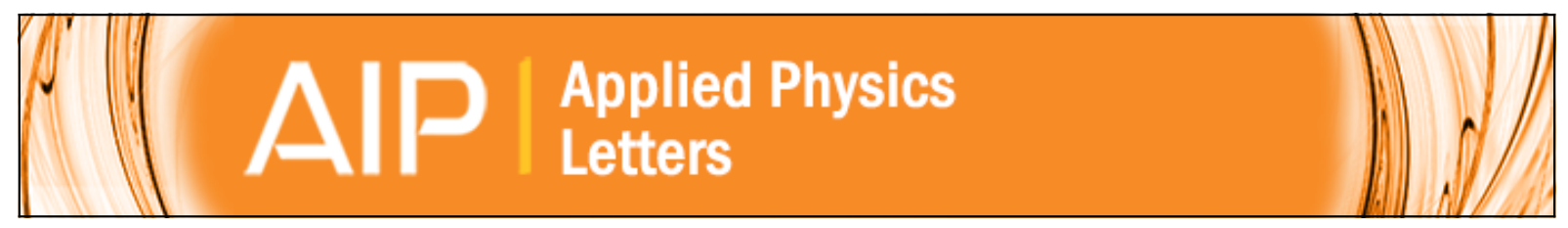

\title{
Dynamics of light-induced FeB pair dissociation in crystalline silicon
}

L. J. Geerligs and Daniel Macdonald

Citation: Applied Physics Letters 85, 5227 (2004); doi: 10.1063/1.1823587

View online: http://dx.doi.org/10.1063/1.1823587

View Table of Contents: http://scitation.aip.org/content/aip/journal/apl/85/22?ver=pdfcov

Published by the AIP Publishing

\section{Articles you may be interested in}

Laser induced lifetime degradation in p-type crystalline silicon

J. Appl. Phys. 111, 114515 (2012); 10.1063/1.4725191

Capability of photoluminescence for characterization of multi-crystalline silicon

J. Appl. Phys. 111, 073504 (2012); 10.1063/1.3699275

Detailed analysis of the microwave-detected photoconductance decay in crystalline silicon

J. Appl. Phys. 104, 104503 (2008); 10.1063/1.3021459

Doping dependence of the carrier lifetime crossover point upon dissociation of iron-boron pairs in crystalline silicon

Appl. Phys. Lett. 89, 142107 (2006); 10.1063/1.2358126

Theoretical and experimental study of charge carrier kinetics in crystalline silicon

J. Appl. Phys. 85, 7741 (1999); 10.1063/1.370579

\section{Model PS-100}

Tabletop Cryogenic Probe Station

\section{Lake Shore CRYOTRONICS}

An affordable solution for a wide range of research 


\title{
Dynamics of light-induced FeB pair dissociation in crystalline silicon
}

\author{
L. J. Geerligs ${ }^{\text {a) }}$ \\ Energy Research Centre of the Netherlands ECN, Solar Energy, P.O. Box 1, NL-1755 ZG Petten, \\ The Netherlands \\ Daniel Macdonald \\ Department of Engineering, FEIT, Australian National University, Canberra, ACT 0200, Australia
}

(Received 23 July 2004; accepted 30 September 2004)

\begin{abstract}
The dynamics of light-induced dissociation of iron-boron (FeB) pairs in $p$-type crystalline silicon is investigated. The dissociation is observed to be a single-exponential process which is balanced with thermal repairing. The dissociation rate is proportional to the square of the carrier generation rate and the inverse square of the $\mathrm{FeB}$ concentration. This suggests that the dissociation process involves two recombination or electron capture events. A proportionality constant of $5 \times 10^{-15} \mathrm{~s}$ describes the dissociation rate well in the absence of other significant recombination channels. The dissociation rate decreases in the presence of other recombination channels. These results can be used for reliable detection of iron in silicon devices and materials, and for further elucidation of the electronically driven FeB dissociation reaction. (C) 2004 American Institute of Physics. [DOI: 10.1063/1.1823587]
\end{abstract}

Iron is an important contaminant in silicon-based integrated circuit and solar cell technology. The presence of interstitial iron $\left(\mathrm{Fe}_{i}\right)$ in silicon can dramatically reduce the nonequilibrium carrier lifetime, even at very low concentrations. Therefore the most sensitive technique available for detecting iron contamination is based on lifetime or diffusion length measurements. ${ }^{1}$ It utilizes the fact that the iron content of a boron-doped $p$-type $\mathrm{Si}$ wafer can be deliberately cycled between being mostly present as $\mathrm{Fe}_{i}$ or as pairs with substitutional boron: $\left(\mathrm{Fe}_{i}\right)^{+}\left(\mathrm{B}_{s}\right)^{-}$pairs. At room temperature in thermal equilibrium virtually all $\mathrm{Fe}_{i}$ is present as $\mathrm{FeB}$ pairs. FeB pair dissociation can be accomplished by illumination at room temperature, ${ }^{2}$ by minority carrier injection, ${ }^{3}$ or by increasing temperature which shifts the equilibrium state of $\mathrm{Fe}_{i}$ and $\mathrm{B}$ to isolated ions. ${ }^{1}$ The isolated and paired form of interstitial iron have markedly different recombination properties. ${ }^{4,5}$ This leads to significant changes in carrier lifetime and diffusion length after dissociating the FeB pairs. Assuming that all other recombination processes remain unchanged, the FeB concentration can be found by measuring the carrier lifetime before $\left(\tau_{0}\right)$ and after $\left(\tau_{1}\right)$ pair dissociation: $[\mathrm{FeB}]=C\left(\tau_{1}^{-1}-\tau_{0}^{-1}\right)$. The use of the known recombination parameters of both $\mathrm{FeB}$ pairs and $\mathrm{Fe}_{i}$ provides the prefactor $C{ }^{6}$ This technique for detection of iron has become widely used in commercial applications for contamination control in microelectronics, as well as being an extremely useful tool for researching iron in silicon and gettering processes in silicon photovoltaics.

The repairing kinetics of $\mathrm{FeB}$ was investigated previously by Zoth and Bergholz ${ }^{1}$ and was reported to follow an exponential behavior with repairing rate $\Gamma_{r}=\left(N_{A} T / 4.3\right.$ $\left.\times 10^{5}\right) \exp \left(0.68 \mathrm{eV} / k_{B} T\right)$ with the boron dopant density $N_{A}$ in $\mathrm{cm}^{-3}$ and $T$ in $\mathrm{K}$. The kinetics of the light-induced dissociation of FeB has not been reported in detail, even though illumination is most often used in practice. It is believed that the process is recombination-enhanced or recombinationinduced. This letter presents experimental results and modeling which describe quantitatively the light-induced dissocia-

${ }^{\text {a)} E l e c t r o n i c ~ m a i l: ~ g e e r l i g s @ e c n . n l ~}$ tion, and provide insight into the dissociation mechanism and the dependence on sample characteristics. The results are compared to earlier carrier-injection driven FeB pair dissociation reported by Kimerling and Benton. ${ }^{3}$ As these authors noted, apart from the technological relevance, the dissociation is in itself an interesting object for study of electronically driven defect reactions.

Details of the investigated samples are given in Table I. Most of the experiments were performed on monocrystalline float-zone (FZ) silicon. Some multicrystalline (mc-Si) samples were also investigated because of the dominance of this material in solar cell technology. The FZ samples were contaminated with $\mathrm{Fe}$ by implantation or indiffusion. ${ }^{4} \mathrm{mc}-\mathrm{Si}$ already contains a significant amount of interstitial $\mathrm{Fe}$ from the growth process and therefore the mc-Si samples did not receive any additional iron. The samples received a surfacepassivating $\operatorname{SiN}_{x}$ coating (surface recombination velocity less than $100 \mathrm{~cm} / \mathrm{s}$ ) and carrier lifetime was measured by quasisteady-state photoconductance (QSSPC). ${ }^{7}$ The total FeB concentration was determined by lifetime change after intense illumination, sufficient to dissociate all $\mathrm{FeB}$ pairs. In two samples the surface recombination rate was deliberately increased, for sample FZ8* by corona charging the $\mathrm{SiN}_{x}$ coating, and for sample $\mathrm{MClc} *$ by omitting the $\mathrm{SiN}_{x}$ coating altogether.

The FeB dissociation was accomplished by illumination with halogen lights, at or slightly below room temperature. Intervals of illumination were alternated with measurements of the momentary FeB concentration. The repairing during each measurement can be calculated and is only significant $(4 \%-10 \%)$ in a few samples with high $[\mathrm{FeB}]$ and high $N_{A}$, in which case the measurements were corrected for this effect. The minority carrier generation rate $G$ due to the illumination was calculated from the illumination level in suns, ${ }^{8}$ as measured with a high-efficiency silicon solar cell. 1 sun was estimated to result in $G=2.1 \times 10^{17} \mathrm{~cm}^{-2} \mathrm{~s}^{-1} / t$, where $t$ is the sample thickness. In samples $\mathrm{FZ1b}$ and $\mathrm{MC} 1 \mathrm{c} *$ the carrier diffusion length was so low that the excess carrier density was inhomogeneous over the thickness of the sample, therefore $t$ was replaced by an effective thickness. ${ }^{9}$ 
TABLE I. Samples investigated in this paper. FZ designates a monocrystalline float-zone silicon sample, MC a multicrystalline silicon sample. All samples apart from $\mathrm{MClc}^{*}$ are surface-passivated with $\mathrm{SiN}_{x}$. MC1c and $\mathrm{MC} 1 \mathrm{c}^{*}$ are sister wafers. $\tau_{\mathrm{other}} / \tau_{\mathrm{FeB}}$ is a measure of the importance of recombination through $\mathrm{FeB}$ vs recombination through other channels. $m_{\mathrm{fit}}$ is the fitted exponent of the dependence of FeB dissociation rate on minority carrier generation rate: $\Gamma_{d} \propto G^{m}$.

\begin{tabular}{lccccc}
\hline \hline \multicolumn{1}{c}{ Sample } & $\rho(\Omega \mathrm{cm})$ & $N_{A}\left(\mathrm{~cm}^{-3}\right)$ & {$[\mathrm{FeB}]\left(\mathrm{cm}^{-3}\right)$} & $\tau_{\text {other }} / \tau_{\mathrm{FeB}}$ & $m_{\text {fit }}$ \\
\hline FZ1a & 1 & $1.5 \times 10^{16}$ & $1.5 \times 10^{11}$ & 1.2 & 1.8 \\
FZ1b & 1.1 & $1.4 \times 10^{16}$ & $1.1 \times 10^{13}$ & 13 & \\
FZ4 & 4 & $3.5 \times 10^{15}$ & $5.4 \times 10^{12}$ & $\gg 10$ & 1.7 \\
FZ8 & 8 & $1.7 \times 10^{15}$ & $6.3 \times 10^{11}$ & 3.4 & $1.5-2.2$ \\
FZ8 & & & & & \\
corona-charged & 8 & $1.7 \times 10^{15}$ & $6.3 \times 10^{11}$ & 1.5 & \\
FZ20 & 20 & $6.8 \times 10^{14}$ & $8 \times 10^{11}$ & $>10$ & 2.8 \\
MC1a & 1.1 & $1.3 \times 10^{16}$ & $2.3 \times 10^{11}$ & 0.8 & \\
MC1b & 0.9 & $1.7 \times 10^{16}$ & $6.9 \times 10^{11}$ & 1.7 & \\
MC1c & 1.2 & $1.3 \times 10^{16}$ & $2.5 \times 10^{12}$ & 10 & 1.6 \\
MC1c* & & & & & \\
unpassivated surface & 1.2 & $1.3 \times 10^{16}$ & $2.5 \times 10^{12}$ & 0.14 & \\
\hline \hline
\end{tabular}

Figure 1 shows FeB dissociation data of sample FZ8 for two illumination intensities, as an example of typical curves. The results are expressed in $n(t) \equiv N_{\mathrm{FeB}}(t) / N_{\mathrm{FeB}}(0)$, i.e., the concentration of $\mathrm{FeB}$ pairs after illumination for a cumulative duration $t$, relative to the total concentration at $t=0$. It can be observed that the FeB concentration initially follows a single-exponential curve, but for large $t$ flattens off to an asymptotic value larger than zero.

This behavior can be described by a single-exponential light-induced $\mathrm{FeB}$ dissociation process with rate $\Gamma_{d}$, balanced with the thermal repairing process with rate $\Gamma_{r}$. If dissociation and repairing rates do not depend on the relative concentrations of $\mathrm{Fe}_{i}$ and $\mathrm{FeB}$, then $d n / d t=-n(t)\left[\Gamma_{r}+\Gamma_{d}\right]+\Gamma_{r}$, and

$$
n(t) \equiv \frac{N_{\mathrm{FeB}}(t)}{N_{\mathrm{FeB}}(0)}=\left[\frac{\Gamma_{d}}{\Gamma_{r}+\Gamma_{d}}\right] e^{-\left(\Gamma_{r}+\Gamma_{d}\right) t}+\frac{\Gamma_{r}}{\Gamma_{r}+\Gamma_{d}} .
$$

Equation (1) was used to fit the data in Fig. 1. It is clear that within measurement accuracy the model describes the data well. For accurate determination of the FeB dissociation

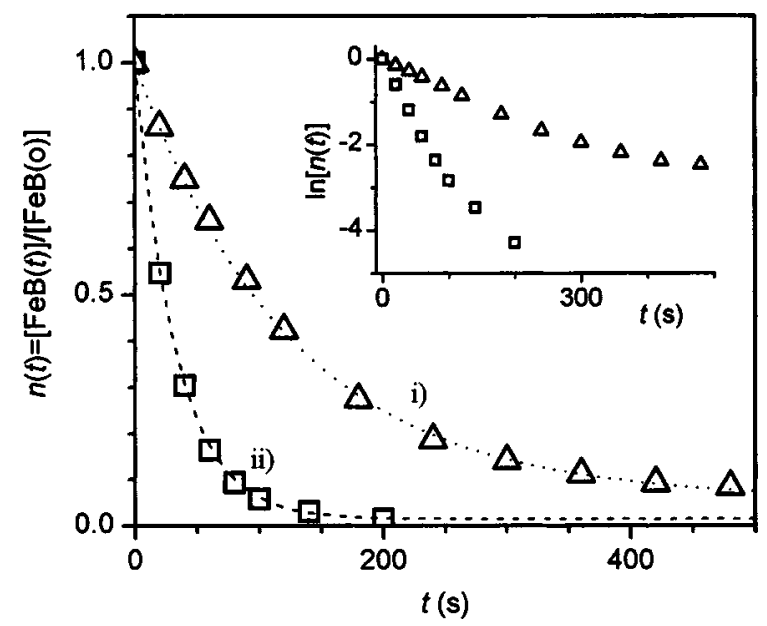

FIG. 1. Normalized FeB concentration as a function of illumination time for sample FZ8, for two different illumination levels: (i) 0.068 sun corresponding to $G=6 \times 10^{17} \mathrm{~cm}^{-3} \mathrm{~s}^{-1}$; and (ii) 0.134 sun corresponding to $G=1.2$ $\times 10^{18} \mathrm{~cm}^{-3} \mathrm{~s}^{-1}$. Inset: logarithmic plot. The dashed and dotted lines are fits to $\mathrm{Eq}$ (1), with $\Gamma_{d^{\prime}}(0.068 \mathrm{sun})=0.0075 \mathrm{~s}^{-1} ; \Gamma_{d}(0.134$ sun $)=0.03 \mathrm{~s}^{-1}$; and $\Gamma_{r}=4.5 \times 10^{-4} \mathrm{~s}^{-1}$. rate $\Gamma_{d}$ for all samples, we have used the fact that the initial decay rate of $\ln [n(t)]$ equals $\Gamma_{d}$, i.e., $(d / d t) \ln [n(t)]_{t=0}=\Gamma_{d}$. Deriving $\Gamma_{d}$ from a fit to the complete $n(t)$ curve is complicated by the fact that for low illumination levels recombination through $\mathrm{Fe}_{i}$ is more effective than through $\mathrm{FeB}$. Thus, as $\mathrm{FeB}$ is being dissociated and replaced by the more recombination-active $\mathrm{Fe}_{i}$, the recombination per $\mathrm{FeB}$ center is reduced. It can therefore be expected that $\Gamma_{d}$ decreases as $n(t)$ is reduced. In agreement with this, we generally found the asymptotic value $n(\infty)=\Gamma_{r} /\left(\Gamma_{r}+\Gamma_{d}\right)$ to be larger than the value calculated from the $t=0$ value of $\Gamma_{d}$ and the independently determined value of $\Gamma_{r}$.

For several samples, $\Gamma_{d}$ was determined as a function of minority carrier generation rate $G$. Those measurements followed a power-law dependence $\Gamma_{d} \propto G^{m}$. Table I lists the fitted value of $m$ for each sample, which varies around $m=2$. Under steady-state conditions, the generation $G$ is balanced by recombination. Recombination in the FZ wafers is dominated by $\mathrm{FeB}$ and $\mathrm{Fe}_{i}$ (at $t=0$, by $\mathrm{FeB}$ only). The scaling of $\Gamma_{d}$ with $G^{m}$, with $m \approx 2$, therefore indicates that two recombination or electron capture events through an FeB center are responsible for the dissociation.

If the $\mathrm{FeB}$ dissociation rate depends on the square of the recombination rate through the $\mathrm{FeB}$ centers, it may then also be expected that, for constant $G$, samples with different FeB concentration show a scaling $\Gamma_{d} \propto[\mathrm{FeB}]^{-2}$. To verify this, in Fig. 2 all measured dissociation rates are plotted as $\Gamma_{d}$ $\times[\mathrm{FeB}]^{2}$ versus carrier generation rate. The FZ sample data in Fig. 2, with the exception of samples FZ1a and FZ20, are indeed quite well arranged on a single line: $\Gamma_{d}=K$ $\times G^{2} /[\mathrm{FeB}]^{2}$, with $K \approx 5 \times 10^{-15} \mathrm{~s}$. This means that the square of the recombination rate per $\mathrm{FeB}$ center determines $\Gamma_{d}$. The cause for the deviation of FZ20 is unclear. The deviation of FZ1a and of the mc-Si samples is attributed to the existence in these samples of significant other recombination channels.

Note that the carrier lifetime is strongly injection-level dependent, and therefore the excess carrier density does not scale linearly with $G$. Scaling of $\Gamma_{d}$ with $G$ is therefore different from scaling with excess carrier density.

If there are other recombination channels (defects, other impurities, surface recombination), the recombination per 


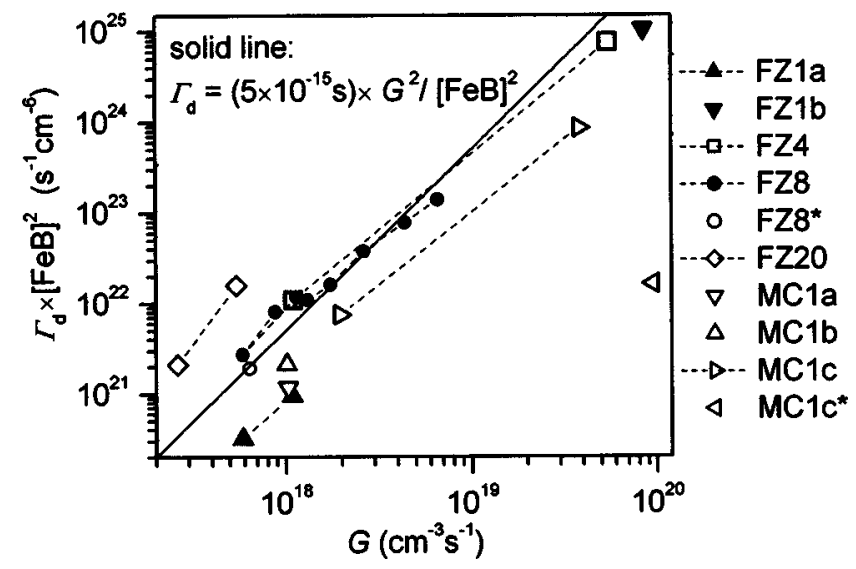

FIG. 2. FeB pair dissociation rates plotted as $\Gamma_{d} \times[\mathrm{FeB}]^{2}$ as a function of light-induced carrier generation $G$. The dashed lines are guides to the eye connecting data points. The solid line shows the quadratic dependence $\Gamma_{d}$ $=\left(5 \times 10^{-15} \mathrm{~s}\right) \times G^{2} /[\mathrm{FeB}]^{2}$.

FeB center is reduced and therefore a lower dissociation rate is expected. We describe the recombination through such other recombination channels by a rate $\tau_{\text {other }}^{-1}$, and the recombination through FeB by the rate $\tau_{\mathrm{FeB}}^{-1}$. Table I shows for each sample the ratio $\tau_{\text {other }} / \tau_{\mathrm{FeB}}$ at an excess carrier density of $10^{15} \mathrm{~cm}^{-3}$. Indeed, when the surface recombination of sample FZ8 was increased by corona-charging of the $\mathrm{SiN}_{x}$, a reduction of the ratio $\tau_{\mathrm{other}} / \tau_{\mathrm{FeB}}$ from 3.4 in FZ8 to 1.5 in FZ8* resulted in a reduction of $\Gamma_{d} \times[\mathrm{FeB}]^{2} / G^{2}$ from 8 to $5 \times 10^{-15} \mathrm{~s}$. Similarly, for samples MC1c and MC1c*, the absence of a surface-passivating coating decreases $\tau_{\mathrm{other}} / \tau_{\mathrm{FeB}}$ from 10 to 0.14 and drastically reduces $\Gamma_{d}$ $\times[\mathrm{FeB}]^{2} / G^{2}$ from $6 \times 10^{-16}$ to $1.8 \times 10^{-18} \mathrm{~s}$. For sample FZ1a, $\tau_{\text {other }} / \tau_{\mathrm{FeB}}=1$ is also relatively low, which may be the reason for the low value of $\Gamma_{d} \times[\mathrm{FeB}]^{2} / G^{2}$ for this sample. With the present data a quantitative analysis of the reduction of $\Gamma_{d}$ due to other recombination channels is however impossible, because $\tau_{\text {other }}$ is determined from QSSPC data at an injection level of $10^{15} \mathrm{~cm}^{-3}$, whereas a precise characterization at the injection levels actually reached during illumination (which are generally much lower) would be necessary. This is difficult to accomplish with the QSSPC method.

The results for the mc-Si samples in Fig. 2 agree well with the FZ samples. It should, however, be noted that in mc-Si samples inhomogeneities affect the results. The QSSPC technique measures the geometrically averaged lifetime. Since low-lifetime areas contribute little to the QSSPC signal, in mc-Si the FeB concentration may be overestimated (we estimate typically by a factor of $\sim 2$ ), 6,10 and the effect of other recombination channels may be underestimated.

Finally, we compare our experiments to earlier reports of carrier-injection induced $\mathrm{FeB}$ pair dissociation, and consider the implications for the dissociation mechanism. Kimerling and Benton studied the kinetics of $\mathrm{FeB}$ dissociation by carrier injection in $n^{+} p$-junctions. ${ }^{3}$ They concluded that a charge-capture driven process was occurring, where neutralization of the $\left(\mathrm{Fe}_{i}\right)^{+}$removes the Coulomb attraction between $\mathrm{Fe}_{i}$ and $\mathrm{B}_{s}$. They noted that by itself this neutralization is not sufficient to result in a significant dissociation rate at room temperature, and also cannot result in the observed temperature dependence with a very low activation energy of $0.1 \mathrm{eV}$.
Therefore, an additional "energy deposition process" was involved in their model. The observation of a quadratic dependence on generation level in our experiments is consistent with this interpretation. The quadratic dependence implies that two electron capture events are involved in FeB dissociation. A possible mechanism may be, for example, that the $\mathrm{Fe}_{i}$ is negatively charged by two capture events, resulting in Coulomb repulsion of the $\mathrm{Fe}_{i} \mathrm{~B}_{s}$ pair. An alternative mechanism may be that one recombination event is the "energy deposition process" and a second electron capture results in neutralization of the $\mathrm{Fe}_{i}$, together resulting in pair dissociation.

Kimerling and Benton observed a linear dependence of the dissociation rate on the injected current density, between 0.1 and $1.5 \mathrm{~A} / \mathrm{cm}^{2}$. This seems to contradict our experimental results. This discrepancy is presently not understood. However, the local injection levels used by Kimerling and Benton were very high and therefore Auger recombination may have masked the quadratic dependence (Auger recombination provides an "other recombination channel" as previously discussed, and increases with injection level). In any case, the results presented here are more directly applicable to light-induced dissociation, which is more widely used than dissociation by current injection.

In conclusion, we have presented results showing that the FeB dissociation rate scales with the square of the carrier generation rate, and the inverse square of the $\mathrm{FeB}$ concentration. The proportionality constant of $5 \times 10^{-15} \mathrm{~s}$ describes the dissociation rate well in the absence of other recombination channels. These results show that the square of the recombination rate per FeB center is the determining factor for the dissociation rate, which may help to elucidate the precise dissociation mechanism.

This work was supported by NOVEM (The Netherlands Agency for Energy and the Environment) and the Australian Research Council. We would like to thank A. R. Burgers for helpful advice on the modeling and Kate McClean for performing some of the measurements. L.J.G. thanks Australia and the Australian National University for their hospitality during a visit in which most of the work was performed.

${ }^{1}$ G. Zoth and W. Bergholz, J. Appl. Phys. 67, 6764 (1990).

${ }^{2}$ K. Graff and H. Pieper, J. Electrochem. Soc. 128, 669 (1981).

${ }^{3}$ L. C. Kimerling and J. L. Benton, Physica B \& C 116B, 297 (1983); L. C. Kimerling, Proceedings of the MRS Annual Meeting, 1980, Boston, MA (North-Holland, Amsterdam, 1980), p. 85.

${ }^{4}$ D. Macdonald, A. Cuevas, and J. Wong-Leung, J. Appl. Phys. 89, 7932 (2001).

${ }^{5}$ A. A. Istratov, H. Hieslmair, and E. R. Weber, Appl. Phys. A: Mater. Sci. Process. 69, 13 (1999).

${ }^{6}$ D. H. Macdonald, L. J. Geerligs, and A. Azzizi, J. Appl. Phys. 95, 1021 (2004).

${ }^{7}$ R. A. Sinton and A. Cuevas, Appl. Phys. Lett. 69, 2510 (1996); URL:www.sintonconsulting.com

${ }^{8}$ sun is the unit of illumination intensity concentration. 1 sun results in the same solar cell current as the standardized $\left(1000 \mathrm{~W} / \mathrm{m}^{2}\right.$, AM1.5) terrestrial solar spectrum. See Handbook of Photovoltaic Science and Engineering, edited by A. Luque and S. Hegedus (Wiley, New York, 2003).

${ }^{9}$ The crossover of the lifetime curves of $\mathrm{Fe}_{i}$ and $\mathrm{FeB}$, which occurs at a well-defined excess carrier density, provides this effective thickness. See Ref. 6.

${ }^{10}$ L. J. Geerligs and D. Macdonald (unpublished). 